\title{
Measuring cognitive load using eye-tracking technology in learning content
}

\author{
Sara Maritza Gutiérrez Rondón, Luis Eduardo Bautista Rojas, María \\ Fernanda Maradei García
}

Published: 30 November 2021

\begin{abstract}
The objective of this study is to determine the effect of a cognitive process called Spatial Contiguity on students engaged in a learning activity. In organizing observed information, cognitive processes beyond attention influence the direction and duration of eye movements. Four volunteers participated in a pilot experiment developed using Eye-tracking to measure cognitive processing of stimuli from learning materials presented in two different formats. Eye-tracking is a tool used to record the eye movements of subjects while performing tasks. The results showed statistically significant differences in cognitive processing measures based on the way learning content is presented.
\end{abstract}

\section{Keywords:}

Eye-tracking; Cognitive load; Learning; Fixations; Saccades.

\section{Introduction}

Learning involves a cognitive process in which an individual obtains and puts knowledge into practice [1] Cognitive load measures have been shown to be an important factor in establishing a relationship between the learning performance of students and their cognitive load as an indicator of their mental efforts. Eyetracking data provides information about eye movements, such as the areas where people focus their attention, the information they ignore, and the objects that bother them [2]. The purpose of the developed experiment is to measure a form of cognitive processing called spatial contiguity [3] in students during a learning activity in which the principle of multimedia learning design is, or is not, implemented. The experiment compares a group of two participants who learned with an integrated content presentation (i.e., words positioned near corresponding graphics) with the second group of two participants who learned with a non-integrated or separate

Gutiérrez Rondón, Sara Maritza.

Universidad Industrial de Santander

Bucaramanga, Colombia.

sara2208474@correo.uis.edu.co

Bautista Rojas, Luis Eduardo.

Universidad Industrial de Santander

Bucaramanga, Colombia.

luis.bautista@correo.uis.edu.co

Maradei García, María Fernanda

Universidad Industrial de Santander

Bucaramanga, Colombia.

mafemar@correo.uis.edu.co content presentation (i.e. words read apart from corresponding graphs). Using Eye-tracking we can take direct measurements of cognitive processing, such as the duration of their eye fixation, the number of fixations and saccade velocity. For this study, we start from the assumption, analyzed by Mayer [4], which indicates that the principle of spatial contiguity establishes that people learn better when presented with words and corresponding images together in a visual scene observed by the student - rather than separately. Therefore, it is inferred that the measure of cognitive processing will find a statistically significant difference between learning from integrated multimedia versus non-integrated content presentation formats; and that, in turn, the objectively measured amount of cognitive processing will be lower for the material presented as integrated content. The results provide two outcomes. First, in quantifying the number of eye fixations and the total time in task development, the group with non-integrated content in learning material yielded higher values. And second, for the measurements of duration of eye fixations and saccade velocity, higher values were associated with learning material presented with integrated content.

\section{Theoretical Background}

\subsection{Cognitive load}

Cognitive load refers to the resources used by working memory and that affect the student while performing a cognitive task [5][6]. According to cognitive load theory, three types of cognitive load interfere with learning: (a) intrinsic load caused by the inherent complexity of instructional information, (b) load directly related to schema construction and automation, and (c) extraneous load caused by instructional elements that are unnecessary for learning [7]. The intrinsic and extraneous load are additive, together they determine the total cognitive load imposed by the learning material, which determines the working memory resources necessary to process the information. Currently, researchers and professionals of visual computing want to reduce the extraneous cognitive load so that most of the working memory resources can be dedicated to learning through the correct design of instructional material [4].

\subsection{Evaluation of cognitive load using eye- tracking}

Objective cognitive load measurement methods are of great importance for research on learning since they measure cognitive load while it occurs [8], that is, while the participant is observing the stimulus, they do not present interruption in the learning processes for the assessment of cognitive activity and load. One of 
the objective methods for evaluating cognitive load is Eye-tracking, which focuses on capturing eye movements [9]. Eye-tracking allows for very detailed analysis, as it provides a deep insight into the processing of human information regarding the allocation of visual attention and cognitive activity in the process and integration of learning information presented in text or image [8].

\subsection{Measurement of cognitive load using eye- tracking}

Eye-tracking allows the identification of fixations, saccades, pupil dilation and blinking. These movements provide evidence of voluntary and open visual attention because the goal of eye movement measurement and analysis is to obtain information about the attentive behavior of the viewer [10]. Moreover, this study will focus on the analysis of fixations and saccades, as these are the basic unit of data for most Eye-tracking analyses [11]. The increase in fixation duration, the number of fixations and saccade movements point to a higher level of cognitive load, which indicates greater effort in processing the learning material and more use of attention resources [12].

\subsubsection{Fixations}

Fixations are voluntary eye movements and correspond to a focused state in which the eye remains immobile for a period, lasting from 200-300 milliseconds to several seconds. The number of fixations means the number of times a user looked at a given area of the stimulus. Duration of fixation relates to the level of cognitive processing with a longer duration of fixation indicating greater tension on working memory [13].

\subsubsection{Saccades}

Saccades are rapid eye movements used to reposition the fovea to a new location in the visual scene [10]. It refers to a switch between two locations, and it usually takes between 30 and 80 milliseconds to complete. It is inferred that measurements of saccade velocity and saccade length can be determined for the purpose of investigating human mental effort since it has been found that a decrease in saccade velocity indicates tiredness and an increase in saccade velocity indicates greater difficulty in the task [13].

\subsection{Spatial Contiguity Principle}

The Spatial Contiguity Principle indicates that students learn better when the corresponding words and pictures are presented close to each other than when they are presented far from each other since students do not have to use cognitive resources to do a large visual search in the scene and are more likely to retain the information presented in the working memory at the moment [3]. The learning material can be presented in different ways, one of them is a version with integrated content, in which the words and images are presented as close as possible or guiding the student from the text to the image or vice versa, so that encourages students to build mental connections with each other. Students do not have to search to find a image that corresponds to the displayed text; therefore, they can dedicate their cognitive resources to active learning processes, including building connections between words and pictures. In a presentation with separated or non-integrated content, words and images are far from each other in the scene, as when text appears in a section of the visual scene and image in a separate section. Therefore, it is inferred that when estimating cognitive processing measures using Eye-tracking for material presented with integrated content, these will be lower than for material with non-integrated content [4].

\section{Materials and Methods}

\subsection{Ethical Implications}

The study requires participants to use a head-mounted Eye-tracking device; however, it is considered non-invasive and the risk to participants is classified as minimal. Each participant received and signed an informed consent document. To preserve the confidentiality of the information, they were previously identified with coded serial numbers.

\subsection{Experiment}

An experimental design was developed, and the elements can be observed in Table 1. The capture of cognitive load data using Eyetracking was proposed in a controlled environment, where the participants were randomly assigned a learning material with a type of content, whether integrated or non-integrated, to measure the load cognitive and make a comparison between both types of content.

Table 1 Experiment Design.

\begin{tabular}{|l|l|}
\hline Participants & 4 participants (4 male) \\
\hline Task & Procedural training activity \\
\hline Stimuli & $\begin{array}{l}\text { Random assignment of stimuli: } \\
\text {-Integrated } \\
\text {-Non-integrated }\end{array}$ \\
\hline $\begin{array}{l}\text { Independent } \\
\text { Variables }\end{array}$ & $\begin{array}{l}\text {-Slide with integrated learning content } \\
\text {-Slide with non-integrated learning }\end{array}$ \\
\hline $\begin{array}{l}\text { Depentent } \\
\text { Variables }\end{array}$ & $\begin{array}{l}\text { Eye-tracking metrics: fixation duration, } \\
\text { number of fixations, saccade rate. }\end{array}$ \\
\hline
\end{tabular}

The proposed study was established as a quasi-experimental research project, it is a prospective, cross-sectional study, and finally, it is proposed with a descriptive scope.

\subsection{Apparatus}

A setup was determined on a computer to display the stimulus as the Eye-tracking data was captured. A head-mounted ocular tracker SMI [14] was used, which can be seen in Figure 1, implemented to allow free mobility of the participants. SMI software was used to set up the experiment and compile the results: Experiment Center and BeGazeTM 3.7.

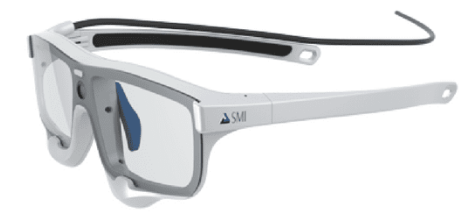

Figure 1 SMI Eye-tracking Glasses

Additionally, the main characteristics of the glasses can be seen in the Table 2 below. 
Table 2 SMI Eye-tracking Glasses characteristics

\begin{tabular}{|l|l|}
\hline Sample rate & $60 \mathrm{~Hz} / 120 \mathrm{~Hz}$ \\
\hline Accuracy & $0.5^{\circ}$ \\
\hline Eye-tracking technique & Dark pupil \\
\hline Eye-tracking type & Binocular \\
\hline Calibration procedure & 0 -point, 1-point and 3-point \\
\hline Scene camera, field of view & $60^{\circ}$ horizontal, $46^{\circ}$ vertical \\
\hline
\end{tabular}

\subsection{Participants}

This study was developed with volunteer participants who had no experience or knowledge related to the capture of measures for the evaluation of cognitive load through Eye-tracking, who were randomly recruited from the Universidad Industrial de Santander. In total, four male subjects between the ages of 21 and 25 participated under the same environmental conditions, the exclusion criteria that were taken into account were to exclude participants who wore glasses or had light eyes. Participants were randomly assigned to Integrated Content or Non-integrated content treatments.

\subsection{Stimuli}

In this study, two stimuli were used which present the learning material in two different forms, where information is provided about the anatomy of the knee and how the perforations of the portals are made to introduce the tools during an arthroscopic intervention of the knee. The first stimulus, which can be seen in Figure 2, is the image in one section and the text in another section, which is called non-integrated content. On the other hand, in Figure 3 we can see the learning material with integrated content, which uses the strategy of placing signs towards the graphic part that describes what the textual part mentions, the integration strategy means placing the graphics and the corresponding words as close as possible to each other.

\subsection{Procedure}

Before the development of the test, each participant is informed about the risks and conditions of the test, and later they were asked to complete a survey about previous knowledge on the learning topic, through a Likert scale, where the score of "1" referred to not having knowledge at all with the subject, and "5" having a lot of knowledge about the subject, this process was done to obtain a homogeneous sample regarding the level of prior knowledge. For this pretest, all of the participants affirmed not having knowledge related to the subject of the learning material, which means they marked "1 I have no knowledge on the subject". During the development of the test, the participant puts on the glasses and the Eye-tracking system was calibrated via three calibration points to which the participant had to direct his gaze and the experimenter had to match the points through a computer; later, the participant is located in front of the computer, where the stimulus assigned randomly is shown on a computer screen and the Eye-tracking data is captured while the participant performs the recognition of the stimulus. The participants did not receive any type of financial reward and took part in the study freely and voluntarily.

\subsection{Hypothesis}

For the hypothesis below, the variables mentioned in Table 1 were taken into account.
- H1: There is a significant difference in the cognitive load measures for the learning material presented with integrated and non-integrated content.
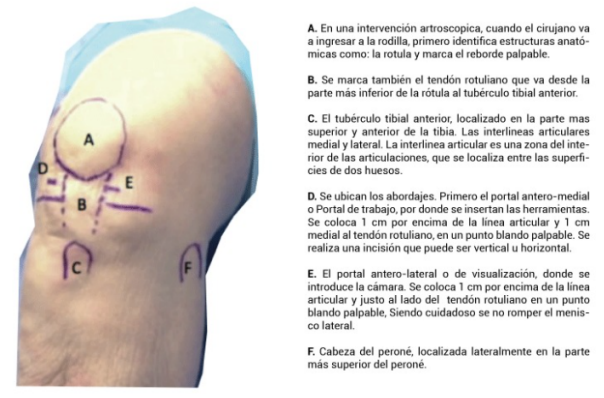

Figure 2 Non-integrated content

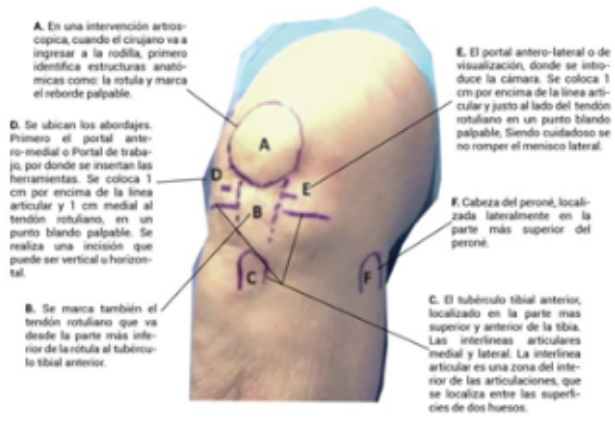

Figure 3 Integrated Content

\subsection{Definition of variables}

\subsubsection{Independent Variables}

As mentioned above, two slides are used with learning material, one with integrated content and the other with non-integrated content.

\subsubsection{Dependent Variables}

- Duration of fixations: Time in which the participant focuses the eye and it remains immobile.

- Number of fixations: Number of times the participant stares at different areas of the stimuli.

- Saccade velocity: Speed of the displacement of the visual focus of attention between one point and another.

\section{Data Analysis and Results}

The experimental design is considered unifactorial since it aims to study the influence of the independent variable on the response variable.

\subsection{Descriptive analysis}

By general inspection of the data, the results shown in Table 3 are observed.

Table 3 Experiment Data

\begin{tabular}{|l|l|l|l|}
\hline Participant & Content & $\begin{array}{l}\text { Total no. of } \\
\text { fixations }\end{array}$ & $\begin{array}{l}\text { Total duration of } \\
\text { experiment [s] }\end{array}$ \\
\hline 1 & $\begin{array}{l}\text { Non- } \\
\text { integrated }\end{array}$ & 678 & 184,30 \\
\hline 2 & $\begin{array}{l}\text { Non- } \\
\text { integrated }\end{array}$ & 585 & 176,90 \\
\hline
\end{tabular}




\begin{tabular}{|l|l|l|l|}
\hline 3 & Integrated & 250 & 77,20 \\
\hline 4 & Integrated & 200 & 62,06 \\
\hline
\end{tabular}

From the data displayed in Table 2, and by calculating the averages of the measurements obtained, it is determined that the average number of fixations and the average of total time implemented for the development of the activity are lower for the material learning with integrated content. Therefore, one possibility of interpretation could be that the integrated learning content requires less mental effort for its execution. However, the data does not show enough evidence to support this claim. An analysis of descriptive statistics related to the duration of the fixations was also developed, where it can be observed that the mean measure is greater for the integrated content with a value of 0.26 [s] compared to the value obtained for the non-integrated content of 0.24 [s], which indicates that the longer the fixations last, there will be a greater effort on the working memory. In the same way, for the data obtained in the descriptive statistics related to the saccade velocity, it can be observed that the mean of the velocity measurement is greater for the integrated content with a value of $61.30[\% \mathrm{~s}]$ compared to the value obtained for the non-integrated content of $54.54[\% \mathrm{~s}]$, which indicates that the faster the saccade velocity, the greater the difficulty in the task. According to the data obtained, it can be interpreted that the visualization of integrated content could generate greater effort concerning the cognitive processing measures.

\subsection{Statistical analysis}

In the first place, for the development of the data analysis, a normality test of the data of Duration of Fixations and Saccade Velocity was developed. According to the analysis developed, the distribution of the data is different from normal. Therefore, the data will be handled as non-parametric, using the Mann Whitney U test and establishing the following statistical hypotheses:

- H0: There are no statistically significant differences in the data $p$-value $>0.05$

- Ha: There are statistically significant differences in the data p-value $<0.05$

For the results of these tests, a $\mathrm{P}=0.0$ is obtained for the duration of the fixations and $\mathrm{P}=0.004$ for the saccade velocity. Because the significance value for both measures of cognitive processing is less than 0.05 , according to this there are statistically significant differences in the values for the integrated and non-integrated content. Finally, a statistically significant difference can be observed for the cognitive processing measures in terms of integrated and non-integrated content, however, this does not present relevant information about which type of content is better to support the learning process.

\section{Discussion}

In a study developed by [15] in which two program codes were analyzed, significant differences were found for the subjective measures of mean duration of fixation and mean saccade amplitude, in two experimental conditions analyzed. The results obtained showed that these values for the objective cognitive load processing measures were associated with the development time of the task, obtaining measures that signified high cognitive load in the experimental conditions that required more time for the development of the activity. Other results of a study [16] analyzed measures based on Eye-tracking, since it was intended to make a comparison between two forms of presentation of learning content, according to the results obtained, the percentage of time spent was the objective measure which was mainly associated with extraneous cognitive load, it was found that the percentage of time spent observing animation by students in a non-redundant condition (low cognitive load) was significantly higher than that of those in a redundant condition (high cognitive load). According to the authors, this means that students invest more attentional resources to observe the animation and establish connections between definitions with a non-redundant condition, instead of using a redundant condition, since there is little time of permanence in the animations that present the lesson demonstrating. Taking into account the approaches made, it is evident that the results of the present study differ from the theories and experiments developed by [3][4][15] and [16], since for these studies a homogeneous behavior of the data is presented regarding the spatial contiguity principle, which presumably ensures that the application to multimedia material leads to cognitive processing measures that indicate a lower cognitive load, than those obtained when the spatial contiguity principle is not applied. This could be due to two aspects; first, the size of the sample, since this was a pilot study and the studies analyzed for the development of this experiment had a minimum sample of 24 participants; second, the nature of the activity, due to the complexity of the task, since, in the studies developed on the subject, tasks with low complexity content are presented, or related to the area of expertise of the students. However, the development of this type of experiment is significant to compare the results obtained and reach a better learning content design, focused on the student having more resources to improve the learning processes.

\section{Conclusion}

In the study developed, there was no evidence that spatial contiguity affected the objective measures of cognitive processing, that is, the presentations of the learning material did not show exact results that indicated a tendency for all the measures of cognitive processing to be lower or higher for one treatment or another since for the treatment with stimulus presented with integrated content, the measurements of the number of fixations and the total time of the experiment are lower than for the treatment with non-integrated content and, on the contrary, concerning the saccade velocity and the duration of the fixations the values obtained are lower for the treatment with non-integrated content than those resulting from the analysis of the integrated content.

\section{Limitations and Future Work}

The experiment developed was limited by the number of participants, which would have been beneficial to have a larger datasheet. One possible alternative interpretation of the results of this study is that the different behaviors of measures of cognitive processing could be attributed to the demands of the task because it was a topic with a high complexity of information, that is, a learning material could have been used of which the students were not aware, but which presented simpler information. Another limitation of the study is that only one set of learning material was used (knee anatomy and description of opening the portals for arthroscopy), so future research is needed to determine if the findings of the cognitive processing measures continue the behavior established in the cognitive theory of multimedia learning and, additionally, if the studies are generalized to other sets of learning materials. 


\section{References}

[1] U. Patayon, J. M. Gallegos, P. Mack, R. Bacabis, and C. Vicente, "Signaling and Pacing: A Comparative Study on Evidence Based Stimuli using an Eye Tracking Device," Procedia Comput. Sci., vol. 179, no. 2019, pp. 313-320, 2021, doi: 10.1016/j.procs.2021.01.011.

[2] D. Mutlu-Bayraktar, P. Ozel, F. Altindis, and B. Yilmaz, "Relationship between objective and subjective cognitive load measurements in multimedia learning," Interact. Learn. Environ., vol. 0, no. 0, pp. 1-13, 2020, doi: $10.1080 / 10494820.2020 .1833042$.

[3] R. E. Mayer, "Spatial Contiguity Principle," Multimed. Learn., pp. 81-95, 2012, doi: 10.1017/cbo9781139164603.006.

[4] C. I. Johnson and R. E. Mayer, "An eye movement analysis of the spatial contiguity effect in multimedia learning," $\mathrm{J}$. Exp. Psychol. Appl., vol. 18, no. 2, pp. 178-191, 2012, doi: 10.1037/a0026923.

[5] F. G. Paas, J. J. Van Merriënboer, and J. J. Adam, "Measurement of cognitive load in instructional research.," Percept. Mot. Skills, vol. 79, no. 1 Pt 2, pp. 419-430, 1994, doi: 10.2466/pms.1994.79.1.419.

[6] L. Perkhofer and O. Lehner, Using gaze behavior to measure cognitive load, vol. 29. Springer International Publishing, 2019.

[7] J. Sweller, J. J. G. van Merriënboer, and F. Paas, "Cognitive Architecture and Instructional Design: 20 Years Later," Educ. Psychol. Rev., vol. 31, no. 2, pp. 261-292, 2019, doi: 10.1007/s10648-019-09465-5.
[8] A. Korbach, R. Brünken, and B. Park, "Measurement of cognitive load in multimedia learning: a comparison of different objective measures," Instr. Sci., vol. 45, no. 4, pp. 515-536, 2017, doi: 10.1007/s11251-017-9413-5.

[9] K. Rayner, Eye movements and attention in reading, scene perception, and visual search, vol. 62, no. 8. 2009.

[10] A. T. Duchowski, Eye Tracking Methodology. 2017.

[11] B. T. Carter and S. G. Luke, "Best practices in eye tracking research,” Int. J. Psychophysiol., vol. 155, no. October 2019, pp. 49-62, 2020, doi: 10.1016/j.ijpsycho.2020.05.010.

[12] M. Keskin, K. Ooms, A. O. Dogru, and P. De Maeyer, "Exploring the Cognitive Load of Expert and Novice Map Users Using EEG and Eye Tracking," ISPRS Int. J. GeoInformation, vol. 9, no. 7, 2020, doi: 10.3390/ijgi9070429

[13] J. Zagermann, U. Pfeil, and H. Reiterer, "Measuring cognitive load using eye tracking technology in visual computing," ACM Int. Conf. Proceeding Ser., vol. 24October, pp. 78-85, 2016, doi: 10.1145/2993901.2993908.

[14] Imotions, "SMI Eye Tracking Glasses," 2021. https://imotions.com/hardware/smi-eye-tracking-glasses/.

[15] M. Andrzejewska and A. Skawińska, "Examining Students' Intrinsic Cognitive Load During Program Comprehension An Eye Tracking Approach," Lect. Notes Comput. Sci. (including Subser. Lect. Notes Artif. Intell. Lect. Notes Bioinformatics), vol. 12164 LNAI, no. Icl, pp. 25-30, 2020, doi: 10.1007/978-3-030-52240-7_5.

[16] T. Zu, J. Hutson, L. C. Loschky, and N. Sanjay Rebello, "Use of eye-tracking technology to investigate cognitive load theory," arXiv, pp. 472-475, 2018, doi: 10.1119/perc.2017.pr.113.

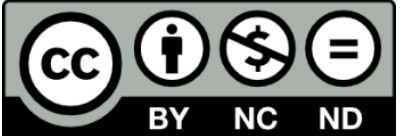

(C) 2021 by the authors. This work is licensed under the Creative Commons AttributionNonCommercial-NoDerivatives 4.0 International License. To view a copy of this license, visit http://creativecommons.org/licenses/by-nc-nd/4.0/ or send a letter to Creative Commons, PO Box 1866, Mountain View, CA 94042, USA. 